\title{
Clinical and epidemiological characteristics of KPC-producing Klebsiella pneumoniae from bloodstream infections in a tertiary referral center in Italy
}

Lucia Brescini ${ }^{1}$, Gianluca Morroni ${ }^{1}$, Chiara Valeriani ${ }^{1}$, Sefora Castelletti ${ }^{1}$, Marina Mingoia ${ }^{2}$, Serena Simoni ${ }^{2}$, Annamaria Masucci ${ }^{3}$, Roberto Montalti ${ }^{4}$, Marco Vivarelli ${ }^{4}$, Andrea Giacometti ${ }^{1}$ and Francesco Barchiesi ${ }^{1,5^{*}}$ (D)

\begin{abstract}
Background: Bloodstream infections (BSI) due to Klebsiella pneumoniae carbapenemase (KPC)-producing K. pneumoniae (KPC-Kp) have become an important problem and they are associated with a high mortality rate. The aim of our study was to evaluate the clinical and epidemiological characteristics of KPC-Kp from BSIs.

Methods: In this retrospective cohort study, conducted in a tertiary referral center in Italy, 112 patients with KPC-Kp BSIs diagnosed between February 2011 and December 2015 were identified. We evaluated the mortality at 30 days from the first positive blood culture. Survivor and non-survivor subgroups were compared to identify predictors of mortality.

Results: The overall crude mortality was 35\%. APACHE II score $\geq 15$, septic shock at BSI onset, immunosuppressive therapy during the 30 days before the BSI onset, and the lack of a combination therapy with at least 2 active drugs emerged as independent predictors of mortality. Excluding patients with inadequate therapy, the mortality decreased to $25 \%$ while an APACHE II score $\geq 15$ and the presence of septic shock remained independently associated with a negative outcome.

Two different pulsotypes were identified: pulsotype A belonged to ST512 and carried KPC-3 and pulsotype B belonged to ST307 and carried KPC-2.

Conclusions: This study confirmed a high mortality rate of KPC-Kp BSIs. The outcome is heavily influenced by the patient's clinical conditions. A therapeutic approach including a combination with at least two active drugs in vitro can improve the prognosis, unless patients received an appropriate therapy.
\end{abstract}

Keywords: Bloodstream infections, Klebsiella pneumoniae, KPC, Colistin resistance

\section{Background}

Bloodstream infections (BSIs) due to Klebsiella pneumoniae carbapenemase (KPC)-producing K.pneumoniae $(\mathrm{KPC}-\mathrm{Kp})$ have become an important problem in the last few years [1-3]. Infections caused by KPC-Kp isolates are associated with a high mortality rate, ranging from 22 to

\footnotetext{
*Correspondence: f.barchiesi@univpm.it

${ }^{1}$ Clinica Malattie Infettive, Dipartimento di Scienze Biomediche e Sanità Pubblica, Università Politecnica delle Marche, Azienda

Ospedaliero-Universitaria Ospedali Riuniti Umberto I'-Lancisi-Salesi, Via Conca, 60126 Ancona, Italy

${ }^{5}$ Malattie Infettive, Azienda Ospedaliera Ospedali Riuniti Marche Nord, Pesaro, Italy

Full list of author information is available at the end of the article
}

$72 \%$. This variability depends on the different characteristics of the populations considered in the studies [2-8].

KPC-Kp strains are endemic in some countries, mainly in the United States, Israel, Latin American countries and Southern Europe [9]. The Annual Report of the European Antibiotic Surveillance Network (EARS-Net), published in 2016, reported a mean percentage of carbapenems resistance equal to $6,1 \%$, with prevalent distribution in Greece, Italy and Romania [10].

Although several studies have demonstrated the efficacy of combination regimens in terms of decreased mortality, an effective treatment is still a challenge for clinicians [9]. Colistin, tigecycline and gentamicin were

(c) The Author(s). 2019 Open Access This article is distributed under the terms of the Creative Commons Attribution 4.0 International License (http://creativecommons.org/licenses/by/4.0/), which permits unrestricted use, distribution, and 
the most widely used antibiotics in these therapeutic regimens but increasing resistance to these drugs has been reported in recent years [11]. In particular, the emergence of colistin resistance among $\mathrm{KPC}-\mathrm{Kp}$ is becoming a major problem. Literature data suggest an increasing mortality in patients infected with colistinresistant (Col-R) KPC-Kp [12-15].

The aim of our retrospective cohort study was to evaluate the clinical and epidemiological characteristics of KPC-producing Klebsiella pneumoniae from bloodstream infections in a tertiary referral center in Italy.

In particular, the mortality at 30 days from the first positive blood culture was considered. Survivor and non-survivor subgroups were compared to identify predictors of mortality.

\section{Methods}

\section{Study setting, data collection and definitions}

The setting was a 980 beds Regional University Hospital in Ancona, Central Italy, including five intensive care units (ICUs), 11 medical and 11 surgical wards. All patients ( $\geq 18$ years old) with BSIs due to KPC-Kp diagnosed between February 2011 and December 2015 were considered. A KPC-Kp BSI was documented by either one or more positive blood culture [16]. BSIs were defined as hospital-acquired if the index blood culture had been collected $>48 \mathrm{~h}$ after hospital admission and no signs or symptoms of infection had been noted at admission.

Patient variables included age, sex, presence of acute or chronic comorbidities, Charlson comorbidity index and APACHE II score, previous surgery ( $\leq 30$ days before BSI onset), any invasive procedures ( $\leq 72 \mathrm{~h}$ before BSI onset), steroid and/or immunosuppressive therapy taken over the previous 30 days, previous antimicrobial therapy $(\leq 30$ days). The isolation of KPC-strains from other sites as well previous ( $\leq 30$ days) or concomitant infections were also considered.

Hospitalization variables included nosocomial or healthcare-related infections, ward submitting index culture, time interval from admission, total days of hospitalization in the previous year.

Treatment variables included empiric antibiotic therapy, defined as treatment administered the same day of first positive blood culture collection (adequate or not, based on subsequent in vitro results), and post-antibiogram therapy (type and number of drugs [i.e.: monotherapy or combination therapy]). Antibiotic therapy was considered adequate if it met two criteria: drug administration within 5 days from BSI onset and the use of at least one drug which was active in vitro. An antibiotic was considered active in vitro when the isolate was susceptible to this specific drug.

The outcome measured was death within 30 days from the first positive blood culture. Survivor and non-survivor subgroups were compared to identify predictors of mortality. Patients who died within $24 \mathrm{~h}$ after the blood culture collection were excluded from the analysis.

\section{Microbiology}

Identification of species was performed with MALDITOF mass spectrometry. Rectal swabs from all patients were collected at admission and screened for the presence of ertapenem-resistant enteric bacteria and main carbapenemases genes. Detection of KPC was assessed with Genexpert (Cepheid, Sunnyvale, CA, USA). KPCKp isolates were genotyped by XbaI-PFGE as previously described [17] and chromosomal DNA restriction patterns were interpreted following the criteria of Tenover et al. [18]. Some representative KPC-Kp isolates for each different PFGE profiles were subjected to MLST analysis [19] and amplification followed by Sanger sequencing of blaKPC genes. The allele sequences and sequence types (STs) were identified at http://bigsdb. pasteur.fr/klebsiella/klebsiella.html.

Susceptibility testing were performed by Vitek 2 system (bio-Merieux, Marcy l'Etoile, France). Colistin MICs were determined by the reference broth microdilution method and results were interpreted according to the EUCAST definition [20].

\section{Statistical analysis}

Categorical variables were expressed as absolute numbers and their relative frequencies; continuous variables were expressed as median and interquartile range (IQR). Categorical variables were compared by the $X^{2}$ or Fisher exact test, while continuous variables were evaluated by the Student $t$ test (for normally distributed variables) or the Mann-Whitney $U$ test (for nonnormally distributed variables). Variables which reached a statistical significance $(p<0.05)$ at univariate analysis were analyzed by multivariate logistic regression analysis to identify independent risk factors for mortality. Kaplan-Meier curves were constructed to compare 30-day survival between patients treated with at least 2 active drugs and those treated with $<2$ active drugs and analyzed by log-rank test. The results obtained were analyzed using the software package SPSS 20.0 (IBM, Armonk, NY, USA).

\section{Results}

During the study period, a total of 112 patients with KPCKp BSIs were identified. Baseline characteristics of the study cohort are reported in Table 1 . The majority were male $(72 \%)$ and had a low performance status (i.e.: $83 \%$ had Charlson Comorbidity Index $\geq 3$ and $50 \%$ had an Apache II score $\geq 15$ ). Cardiovascular and neurological diseases along with solid tumors were the most common underlying diseases $(54,34,33 \%$, respectively). Septic shock was present in $36 \%$ of the overall population at the BSI onset. The highest proportion of patients was hospitalized in 
Table 1 Demographic and clinical characteristics of the study cohort

\begin{tabular}{|c|c|c|c|c|}
\hline \multirow[t]{2}{*}{ Variables } & \multicolumn{4}{|l|}{ 30-days outcome } \\
\hline & All, 112 & Death, 39 (35\%) & Survive, 73 (65\%) & $p$-value \\
\hline \multicolumn{5}{|l|}{ Patients variables } \\
\hline \multicolumn{5}{|l|}{ Sex } \\
\hline - Male & $80(72 \%)$ & $27(69 \%)$ & $53(73 \%)$ & \multirow[t]{2}{*}{0.875} \\
\hline - Female & $32(28 \%)$ & $12(31 \%)$ & $20(27 \%)$ & \\
\hline Age (years) median (IQR) & $68(55-76)$ & $72(58-78)$ & $65(54-75)$ & 0.528 \\
\hline Charlsons Comorbidity Index $\geq 3$ & $93(83 \%)$ & $37(95 \%)$ & $56(77 \%)$ & 0.030 \\
\hline \multicolumn{5}{|l|}{ Comorbidities } \\
\hline - Diabetes & $31(28 \%)$ & $9(23 \%)$ & $22(30 \%)$ & 0.566 \\
\hline$\cdot$ COPD & $18(16 \%)$ & $7(18 \%)$ & $11(15 \%)$ & 0.900 \\
\hline - Haematological malignancies & $16(14 \%)$ & $9(23 \%)$ & $7(10 \%)$ & 0.097 \\
\hline - Solid tumors & 37 (33\%) & $15(39 \%)$ & $22(30 \%)$ & 0.496 \\
\hline - Chronic Hepatitis & $10(9 \%)$ & $5(13 \%)$ & $5(7 \%)$ & 0.313 \\
\hline - Cardiovascular disease & $66(54 \%)$ & $23(59 \%)$ & $43(59 \%)$ & 0.994 \\
\hline - Neurological disease & $38(34 \%)$ & $6(15 \%)$ & $32(44 \%)$ & 0.005 \\
\hline - Chronic kidney disease & 32 (29\%) & $14(36 \%)$ & $18(25 \%)$ & 0.301 \\
\hline$\cdot \mathrm{HIV}$ & $1(1 \%)$ & 0 & $1(1 \%)$ & 1.000 \\
\hline - Neutropenia & $12(11 \%)$ & $8(21 \%)$ & $4(6 \%)$ & 0.033 \\
\hline - Gastrointestinal disease & $23(21 \%)$ & $5(13 \%)$ & $18(25 \%)$ & 0.218 \\
\hline$\cdot$ SOT & $5(5 \%)$ & $3(8 \%)$ & $2(3 \%)$ & 0.226 \\
\hline - Others & $21(19 \%)$ & $5(13 \%)$ & $16(22 \%)$ & 0.357 \\
\hline Apache II score $\geq 15$ & $56(50 \%)$ & $32(82 \%)$ & $24(33 \%)$ & $<0.001$ \\
\hline \multicolumn{5}{|l|}{ Acute comorbidities } \\
\hline - Septic shock & $40(36 \%)$ & $26(67 \%)$ & $14(19 \%)$ & $<0.001$ \\
\hline - pneumonia & $48(43 \%)$ & $21(54 \%)$ & $27(37 \%)$ & 0.129 \\
\hline - Acute kidney failure & $18(16 \%)$ & $8(20 \%)$ & $10(14 \%)$ & 0.506 \\
\hline - Gastrointestinal perforation & $5(5 \%)$ & $4(10 \%)$ & $1(1 \%)$ & 0.049 \\
\hline - Trauma & $10(9 \%)$ & $2(5 \%)$ & $8(11 \%)$ & 0.489 \\
\hline - Others & $53(47 \%)$ & $16(41 \%)$ & $37(51 \%)$ & 0.329 \\
\hline \multicolumn{5}{|l|}{ Hospitalization variables } \\
\hline Nosocomial infection & 104 (93\%) & $36(92 \%)$ & $68(93 \%)$ & 1.000 \\
\hline Healthcare-related infection ${ }^{a}$ & $8(7 \%)$ & $3(8 \%)$ & $5(7 \%)$ & \\
\hline \multicolumn{5}{|l|}{ Wards submitting index culture } \\
\hline - Intensive care unit & $45(40 \%)$ & $15(38 \%)$ & $30(41 \%)$ & 0.945 \\
\hline - Surgery & $27(24 \%)$ & $7(18 \%)$ & $20(27 \%)$ & 0.378 \\
\hline - Medicine & $32(29 \%)$ & $14(36 \%)$ & $18(25 \%)$ & 0.301 \\
\hline - Other health care facilities & $8(7 \%)$ & $3(8 \%)$ & $5(7 \%)$ & 1.000 \\
\hline Time interval (days) from admission, median (IQR) & $23,5(11-39)$ & $23,5(11-39)$ & $24,5(11,5-39)$ & 0.541 \\
\hline Total previous hospitalization, median (IQR) ${ }^{\mathrm{b}}$ & $30,5(15-59.25)$ & $33(15-66)$ & $27(14.5-51.5)$ & 0.352 \\
\hline \multicolumn{5}{|l|}{ Pre-infection variables } \\
\hline Central venous catheter & 99 (88\%) & $39(100 \%)$ & $60(82 \%)$ & 0.004 \\
\hline Other devices & $102(91 \%)$ & $38(97 \%)$ & $64(88 \%)$ & 0.161 \\
\hline $\mathrm{CWH}$ & $8(7 \%)$ & $3(8 \%)$ & $5(7 \%)$ & 1.000 \\
\hline Invasive procedures ${ }^{c}$ & 31 (28\%) & 17 (44\%) & 14 (19\%) & 0.011 \\
\hline
\end{tabular}


Table 1 Demographic and clinical characteristics of the study cohort (Continued)

\begin{tabular}{|c|c|c|c|c|}
\hline \multirow[t]{2}{*}{ Variables } & \multicolumn{4}{|c|}{ 30-days outcome } \\
\hline & All, 112 & Death, 39 (35\%) & Survive, 73 (65\%) & $p$-value \\
\hline Steroid therapy ${ }^{d}$ & $43(38 \%)$ & $18(46 \%)$ & $25(34 \%)$ & 0.303 \\
\hline Immunosuppressive therapy ${ }^{d, e}$ & $29(26 \%)$ & $15(39 \%)$ & $14(19 \%)$ & 0.046 \\
\hline Previous Surgery ${ }^{f}$ & $60(54 \%)$ & $22(56 \%)$ & $51(70 \%)$ & 0.090 \\
\hline - Gastrointestinal surgery & $30(27 \%)$ & $11(28 \%)$ & $19(26 \%)$ & 0.981 \\
\hline - Cardiovascular surgery & $9(8 \%)$ & $5(13 \%)$ & $4(6)$ & 0.272 \\
\hline - Urologic surgery & $7(6 \%)$ & $3(8 \%)$ & $4(6 \%)$ & 0.693 \\
\hline - Neurosurgery & $13(12 \%)$ & $2(5 \%)$ & $11(15 \%)$ & 0.214 \\
\hline - Orthopedic surgery & $9(8 \%)$ & $1(3 \%)$ & $8(11 \%)$ & 0.158 \\
\hline • Plastic surgery & $3(3 \%)$ & 0 & $3(4 \%)$ & 0.550 \\
\hline - Thoracic surgery & $3(3 \%)$ & $1(3 \%)$ & $2(3 \%)$ & 1.000 \\
\hline \multicolumn{5}{|l|}{ Microbiologic variables } \\
\hline KPC rectal swab ${ }^{9}$ & $32(29 \%)$ & $16(41 \%)$ & $16(22 \%)$ & 0.056 \\
\hline \multicolumn{5}{|l|}{ Isolation of KPC from other sites } \\
\hline - Urinary tract & $30(27 \%)$ & $7(18 \%)$ & $23(32 \%)$ & 0.187 \\
\hline - Bronchial / pleural fluid & $36(32 \%)$ & $11(28 \%)$ & $25(34 \%)$ & 0.660 \\
\hline • abdominal fluid & $13(9 \%)$ & $6(15 \%)$ & $7(10 \%)$ & 0.370 \\
\hline - wounds & $15(13 \%)$ & $6(15 \%)$ & $9(12 \%)$ & 0.872 \\
\hline \multicolumn{5}{|l|}{ Other infections, $\mathrm{n}^{\circ}(\%)$} \\
\hline - Previous infections ${ }^{\mathrm{h}}$ & $35(31 \%)$ & $11(28 \%)$ & $24(33 \%)$ & 0.769 \\
\hline - Concomitant & $26(23 \%)$ & $10(26 \%)$ & $16(22 \%)$ & 0.834 \\
\hline \multicolumn{5}{|l|}{ Treatment variables } \\
\hline Previous antibiotic therapy ${ }^{d}$ & $91(81 \%)$ & $33(85 \%)$ & $58(80 \%)$ & 0.680 \\
\hline - Penicillins & $34(30 \%)$ & $12(31 \%)$ & $22(30 \%)$ & 1.000 \\
\hline - Cephalosporins & $8(7 \%)$ & $1(3 \%)$ & $7(10 \%)$ & 0.258 \\
\hline - Carbapenems & $51(46 \%)$ & $20(51 \%)$ & $31(43 \%)$ & 0.488 \\
\hline - Fluoroquinolones & $32(29 \%)$ & $12(31 \%)$ & $20(27 \%)$ & 0.875 \\
\hline - Macrolides & $4(4 \%)$ & 0 & $4(6 \%)$ & 0.296 \\
\hline - Aminoglycosides & $10(9 \%)$ & $1(1 \%)$ & $9(12 \%)$ & 0.161 \\
\hline • Tigecycline & $21(19 \%)$ & $8(21 \%)$ & $13(18 \%)$ & 0.924 \\
\hline - Colistin & $14(13)$ & $6(15)$ & $8(11 \%)$ & 0.708 \\
\hline - Others & $47(42 \%)$ & $19(48 \%)$ & $28(38 \%)$ & 0.391 \\
\hline Adequate empiric antibiotic treatment & $15(13 \%)$ & $6(15 \%)$ & $9(12 \%)$ & 0.872 \\
\hline \multicolumn{5}{|l|}{ Post-antibiogram therapy } \\
\hline - Colistin-including therapy & $44(39 \%)$ & $11(28 \%)$ & $33(45 \%)$ & 0.121 \\
\hline • Tigecycline-including therapy & $57(51 \%)$ & $16(41 \%)$ & $41(56 \%)$ & 0.184 \\
\hline - Gentamicin-including therapy & $46(41 \%)$ & $9(23 \%)$ & $37(51 \%)$ & 0.009 \\
\hline • Monotherapy & $13(12 \%)$ & $3(8 \%)$ & $10(14 \%)$ & 0.537 \\
\hline - Combination therapy & $89(80 \%)$ & $29(74 \%)$ & $60(82 \%)$ & 0.328 \\
\hline - Two-drug combinations & $24(21 \%)$ & $10(26 \%)$ & $14(19 \%)$ & 0.581 \\
\hline - Combinations with $\geq$ three drugs & $66(59 \%)$ & $19(49 \%)$ & $47(64 \%)$ & 0.160 \\
\hline - Carbapenem-excluding combinations & $41(37 \%)$ & $16(41 \%)$ & $25(34 \%)$ & 0.615 \\
\hline - Carbapenem-including combinations & $71(63 \%)$ & $23(59 \%)$ & $48(66 \%)$ & \\
\hline - Double-carbapenem combinations & $5(5 \%)$ & $2(5 \%)$ & $3(4 \%)$ & 1.000 \\
\hline
\end{tabular}


Table 1 Demographic and clinical characteristics of the study cohort (Continued)

\begin{tabular}{|c|c|c|c|c|}
\hline \multirow[t]{2}{*}{ Variables } & \multicolumn{4}{|c|}{ 30-days outcome } \\
\hline & All, 112 & Death, 39 (35\%) & Survive, 73 (65\%) & $p$-value \\
\hline - Rifampin addition to combination & $3(3 \%)$ & $2(5 \%)$ & $1(1 \%)$ & 0.240 \\
\hline - Adequate antibiotic treatment ${ }^{j}$ & $84(74 \%)$ & $21(54 \%)$ & $63(85 \%)$ & $<0.001$ \\
\hline -with one active drug & 49 (44\%) & $17(44 \%)$ & $32(44 \%)$ & 1.000 \\
\hline -with two or three active drugs & $35(31 \%)$ & $4(10 \%)$ & $31(42 \%)$ & $<0.001$ \\
\hline \multicolumn{5}{|l|}{$\mathrm{KPC}-\mathrm{K}$ isolate characteristics } \\
\hline Colistin resistant & $66(59 \%)$ & $22(56 \%)$ & $44(60 \%)$ & 0.846 \\
\hline Tigecycline resistant ${ }^{k}$ & $27(37 \%)$ & $10(45 \%)$ & $17(33 \%)$ & 0.436 \\
\hline Gentamicin resistant & $14(13 \%)$ & $5(13 \%)$ & $9(12 \%)$ & 1.000 \\
\hline Amikacin resistant & $97(86 \%)$ & $33(85 \%)$ & $64(88 \%)$ & 0.872 \\
\hline Fosfomycin resistant & $72(64 \%)$ & $19(49 \%)$ & $53(73 \%)$ & 0.188 \\
\hline Bactrim resistant & $92(82 \%)$ & $30(77 \%)$ & $62(85 \%)$ & 0.426 \\
\hline Meropenem MIC $\leq 8$ & $4(4 \%)$ & $3(8 \%)$ & $1(1 \%)$ & 0.086 \\
\hline Meropenem MIC $\geq 16$ & 108 (96\%) & $36(87 \%)$ & 72 (99\%) & \\
\hline Pulsotype A - ST512 & $97(91 \%)$ & $33(89 \%)$ & $64(91 \%)$ & 0.705 \\
\hline Pulsotype B - ST307' & $10(9 \%)$ & $4(11 \%)$ & $6(9 \%)$ & \\
\hline
\end{tabular}

Data are expressed as No. (\%) unless otherwise specified

Abbreviations: APACHE Acute Physiology and Chronic Health Evaluation, IQR interquartile range, COPD Chronic obstructive pulmonary disease, SOT solid organ transplantation, CVVH Continuous Veno-Venous Hemofiltration

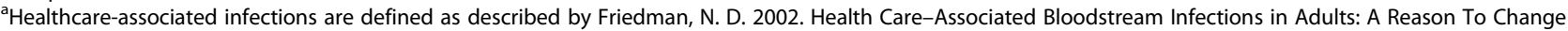
the Accepted Definition of Community-Acquired Infections. Annals of Internal Medicine, 137 (10),

791. doi:https://doi.org/10.7326/0003-4819-137-10-200211190-00007

b During the 12 months preceding BSI onset

'During the $72 \mathrm{~h}$ preceding $\mathrm{BSI}$ onset

${ }^{\mathrm{d}}$ During the 30 days preceding BSI onset

Excluding therapy with steroids

fDuring the 3 months preceding BSI onset

${ }^{9}$ The majority of rectal swabs was collected before the onset of KPC-Kp BSI, during of the same hospitalization

${ }^{h}$ During the 30 days preceding BSI onset. Previous infections included: lung infections $(n=16)$, BSIs $(n=5)$, SSTIs $(n=3)$, CNS infections $(n=2)$, abdominal

infections $(n=2)$, UTIs $(n=2)$, abdominal infection plus BSI $(n=1)$, UTI plus BSI $(n=1)$, lung infection plus BSI ( $n=1)$, lung infection plus SSTI $(n=1)$, lung infection plus UTI $(n=1)$

'Concomitant infections included lung infections $(n=8)$, BSIs $(n=4)$, UTIs $(n=4)$, SSTIs $(n=2)$, abdominal infection ( $n=1)$, BSIs plus lung infections ( $n=3)$, BSI

plus lung infection plus CNS infection plus SSTI $(n=1)$, abdominal infection plus BSI $(n=1)$, lung infection plus UTI ( $n=1)$ and BSI plus UTI $(n=1)$. 10 BSI were polymicrobial

${ }^{j}$ Adequate therapy is defined as the use of at least one drugs to which the isolate was susceptible in vitro

${ }^{\mathrm{k}}$ Strains showing tigecycline MIC $\geq 4 \mathrm{mg} / \mathrm{L}$ were considered non susceptible as stated by Marchaim et al. 2014. Major variation in MICs of tigecycline in Gramnegative bacilli as a function of testing method. J Clin Microbiol, 52:1617-21. doi:https://doi.org/10.1128/JCM.00001-14

'Percentage of these variables are calculated only on the 107 patients from which bacterial strains were recovered

ICUs (40\%). Almost all patients (88\%) carried a central venous catheter (CVC). A high proportion of patients was undergoing steroid or immunosuppressive therapies. Gastrointestinal surgery was the most common type of intervention characterizing these patients.

Ninety-six \% of KPC-Kp demonstrated full resistance to meropenem (MICs $\geq 16 \mu \mathrm{g} / \mathrm{ml}$ ) while the other strains (4\%) were meropenem non-susceptible showing MICs of $8 \mu \mathrm{g} /$ $\mathrm{ml}$. Resistance rates of amikacin and trimethoprimsulfamethoxazole were also high ( 86 and $82 \%$, respectively) while gentamicin was the most active in vitro antibiotic (13\% of isolates were resistant). Notably, resistance to colistin was found in $59 \%$ of the isolates. Colistin-resistant characterized all strains (100\%) isolated in 2011 and 2012 while it decreased in the following years (47\% in 2013 and 2014 and $50 \%$ in 2015).
Previous carbapenem therapy occurred in $46 \%$ of the patients. Empiric antibiotic treatment was considered adequate only in $13 \%$ of the overall population. Post-antibiogram therapy consisted of a combination regimen in $80 \%$ of the cases with a carbapenemincluding approach being the most frequently utilized. Antibiotic treatment was considered adequate in $74 \%$ of the patients: therapy was administered with only one active drug or with two or three active drugs in 44 and $31 \%$ of cases, respectively.

Thirty-day mortality rate was $35 \%$. A significantly high proportion of patients dying within 30 days had a Charlson Comorbidity Index $\geq 3$, an APACHE II score $\geq 15$, neutropenia, septic shock, gastrointestinal perforation, carried a CVC, underwent an invasive procedure within $72 \mathrm{~h}$ and received immunosuppressive therapy ( $\mathrm{p}$ ranging 
from $<0.001$ to 0.049 ). There was a trend, although no statistically significant $(p=0.056)$, of positive KPC rectal swabs in non-survival patients, collected before the BSI onset. On the contrary, survivors most frequently received combination therapy with at least two active drugs $(p<$ $0.001)$ and gentamicin-including regimens in the postantibiogram phase $(p=0.009)$.

In the multivariate logistic regression analysis, APACHE II score $\geq 15$, septic shock at BSI onset, immunosuppressive therapy during the 30 previous days, and the lack of a combination therapy with at least 2 active drugs emerged as independent predictors of mortality (Table 2).

Survival curves confirmed the reduced mortality risk associated with a combination regimen containing at least 2 active drugs compared to other groups of treatment ( $p=0.0006$, Fig. 1$)$.

We performed a further analysis by excluding those patients $(n=28)$ not having received an adequate therapy. This subgroup of patients showed a 30-day mortality of $25 \%$. Independent risk factors for mortality were APACHE II score $\geq 15$ (OR 30.241; 95\% CI, 4.163 to 219.700; $p=$ 0.001), septic shock (OR 6.043; 95\% CI, 1.096 to 33.303; $p=0.039)$ and hospitalization in internal medicine wards (OR 7.299; 95\% CI, 1.174 to 45.376; $p=0.033$ ).

A total of 107 out of 112 isolates were molecularly identified. XbaI-PFGE disclosed two different pulsotypes: the most common was the A pulsotype (97/107 isolates) while B pulsotype was less diffused (10/107 isolates). Ten representative strains from pulsotype A underwent MLST and all belonged to ST512 and harboured KPC-3, differently the 4 representative isolates with pulsotype B that belonged to ST307 and carried KPC-2. Notably, ST307 strains were all susceptible to colistin. We did not find any correlation between 30-day mortality and sequence type.

\section{Discussion}

In this study we evaluated 30-day mortality in patients with BSIs due to KPC-Kp. We found a crude mortality of $35 \%$.
These data are consistent with previous reports, in which the mortality rate ranged from 22 to $72 \%[3,4,7,8]$. This wide variation in mortality can be due to several factors including patient characteristics, type and timing of antibacterial therapy as well resistance patterns of the isolates. In the present study, in which up to $90 \%$ of the isolates had meropenem MIC $>16 \mu \mathrm{g} / \mathrm{ml}$, we did not find any beneficial effect of a carbapenem-containing regimen on survival. Our data agreed with those reported in the literature suggesting that efficacy of meropenem-containing regimens decreased when meropenem MICs were $>8 \mu \mathrm{g} / \mathrm{ml}[2-4]$.

One study evaluated the achievement of pharmacokinetic/pharmacodynamics (PK/PD) targets of meropenem, administered as an extended 3-h infusion of $2 \mathrm{~g}$ every $8 \mathrm{~h}$ in 19 critically-ill patients with KPC-Kp BSIs. As expected, PK/PD target was not achieved when meropenem MICs ranged from 256 to $1024 \mu \mathrm{g} / \mathrm{ml}$ [21]. On the other hand, one recent study showed that high-dose continuous infusion of meropenem, in combination with other antimicrobials with activity against Gram-negative bacteria, was effective in the treatment of KPC-Kp infections caused by isolates with meropenem MICs $\geq 64 \mu \mathrm{g} /$ $\mathrm{ml}$ [22]. Overall, these data indicate that there are still conflicting results on the use of carbapenem-containing regimens when meropenem MIC is high. The recent introduction of new antimicrobial therapies (i.e.: 3rd generation cephalosporin/carbapanems associated with innovative lactamase inhibitors) will overcame this issue.

We found an extremely high resistance rate to colistin (59\%). However, we did not observe a difference in mortality between patients infected with colistinsusceptible and Col-R isolates. Several studies showed a relationship between Col- $\mathrm{R}$ isolates and mortality [12, 15]. In particular, a multicenter, retrospective study conducted in six Italian hospitals, showed a colistin resistance equal to $20 \%$ [12]. Mortality rate in this subgroup of patients was significantly higher than that reported for patients infected with colistin-susceptible isolates ( $51 \%$ vs $39 \%$, respectively). The same study also

Table 2 Multivariate analysis of risk factors for mortality in the study cohort

\begin{tabular}{lll}
\hline Variable & Adjusted OR (95\% Cl) & $p$ \\
\hline All patients $(n=112)$ & & $<0.001$ \\
Apache score $\| \geq 15$ & $12.260(3.653-41.146)$ & 0.001 \\
Septic shock & $6.542(2.209-19.374)$ & 0.025 \\
Immunosoppressive therapy & $4.363(1.201-15.858)$ & 0.014 \\
Antibiotic treatment with & $5.063(1.382-18.546)$ & \\
$<2$ active drugs & & 0.001 \\
Adequate therapy $(n=84)$ & $30.241(4.163-219.700)$ & 0.039 \\
APACHE II score $\geq 15$ & $6.043(1.096-33.303)$ & 0.033 \\
Septic shock & $7.299(1.174-45.376)$ & \\
Hospitalization in internal medicine wards & \\
\hline
\end{tabular}




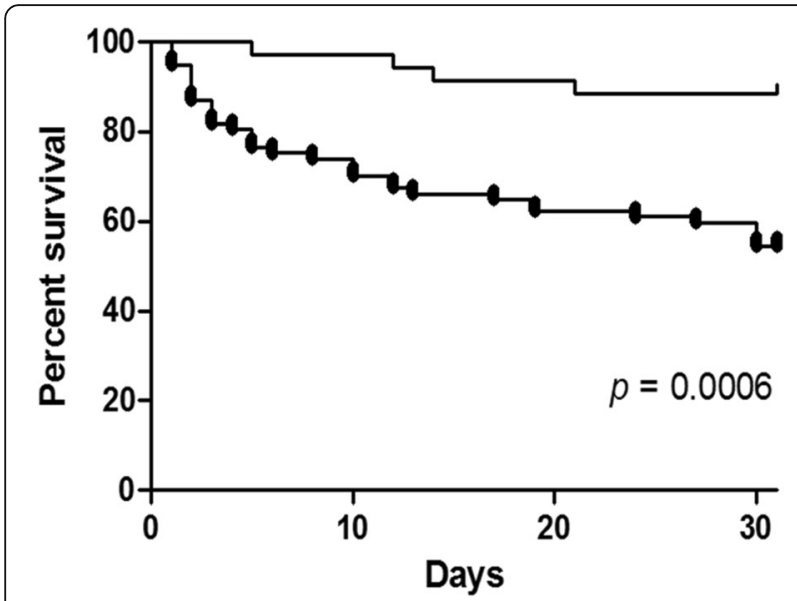

Fig. 1 Kaplan Meier survival curves of patients treated with $\geq 2$ active drugs (flat line) vs those patients treated with $<2$ active drugs (dots line)

noted an increasing prevalence over time of Col- $\mathrm{R}$ $\mathrm{KPC}-\mathrm{Kp}$ and an association between colistin resistance and previous colistin therapy [12]. In contrast with this findings and other data available in the literature [2, 11, $12,15]$, we found a decrease of Col-R KPC-Kp isolates during the study period. This phenomenon can be due to, at least, two factors: the appearance of a new clone susceptible to colistin in 2014 (ST307) and the progressive implementation in our center of the antimicrobial stewardship with significant reduction of colistin use in clinical practice.

Independent risk factors associated with increased mortality in our cohort of patients were an APACHE II score $\geq 15$, the presence of septic shock, the immunosuppressive therapy, and the lack of a combination therapy with at least two active drugs. The first two factors were repeatedly reported in previous studies [2, 3, 13] and emphasize how the patient's clinical status is of paramount importance in determining the outcome of the infection. Although the presence of a current immunosuppressive therapy theoretically correlates well with a negative outcome of the infection, one study found that an immunosuppression status unexpectedly correlated with a better outcome [23]. These paradoxical results have been explained by the exclusion of patients highly likely to have poor outcome (i.e.: too short antibiotic therapy $[\leq 48 \mathrm{~h}]$ and the presence of polymicrobial bacteriemia). Other retrospective studies reported a positive association between combination therapy and 30-day survival $[3,4]$. Although we found that the number of agents with activity in vitro (at least 2 active drugs) is an important factor to guarantee a positive outcome, contrarily to other studies, we did not observe any correlation between the type of therapeutic association and outcome [9]. A possible bias could include the fact that patients who live longer were more likely to received two active drugs. In order to minimize this effect, we performed a sub-analysis excluding patients that have not received an adequate therapy; the results obtained were slightly different. Indeed, while APACHE II score $\geq 15$ and the presence of septic shock remained independent risk factors associated with increased mortality, hospitalization in internal medicine wards emerged as negative prognostic factor. Moreover, in this sub-group the combination therapy disappeared as a positive prognostic factor. These results may be due to the fact that patients received an appropriate drug, the outcome is heavily influenced by the patient's clinical conditions as well as an intensive care unit stay might yield a more adequate support. The majority of KPC-Kp isolated in our hospital belonged to ST512 and produced KPC-3. This finding was not surprising taking into account that most of hospital-associated $\mathrm{KPC}-\mathrm{Kp}$ recovered in Italy belongs to this clonal lineage as reported by a recent national study [24]. The other clone ST307 was already recovered in Italy [24] and belongs to a novel widespread lineage that could emerge as a new clinically relevant clone [25]. It's interesting to note that in our center, KPC-Kp ST307 clone appeared in the early 2014 being isolated in a patient coming from a long-term care facility and it was responsible of a mini-outbreak in several wards. This finding emphasizes how hospital outbreaks could be supported by external clones reaching the hospital and highlights the importance of surveillance also in non-hospital structures.

The present study has some limitations. First, being a retrospective observational study our sample size is too small to allow us to detect subtle differences in treatment outcome. Second, since our data come from a single center experience, our findings may not be relevant to other patients population. Third, although we have made every attempt to collect and analyze as many as clinical data as possible to reveal useful information for patient management, some variables could not be explored because of missing data. Fourth, since EUCAST breakpoints are different from those of CLSI, some results may be different if the CLSI breakpoints are applied. Finally, our study did not include newer agents (e.g., ceftazidime-avibactam, meropenem-vaborbactam) and it is unknown whether combination therapy involving these agents would have been associated with improved outcomes.

\section{Conclusions}

This study confirmed a high mortality rate of KPC-Kp BSIs. The outcome is heavily influenced by the patient's clinical conditions. A therapeutic approach including a combination with at least two active drugs in vitro can improve the prognosis, unless patients received an appropriate therapy. Additional data are needed to further elucidate this finding especially in light of the introduction of the new molecules. 


\section{Abbreviations}

APACHE: Acute Physiology and Chronic Health Evaluation; BSI: Blood stream infections; Cl: Confidence interval; Col-R: Colistin resistant; COPD: Chronic obstructive pulmonary disease; CVC: Central venous catheter; CWH: Continuous Veno-Venous Hemofiltration; HCC: Hepatocellular carcinoma; ICU: Intensive care unit; IQR: Interquartile range; KP: Klebsiella pneumoniae; KPC: Klebsiella pneumoniae carbapenemase; MALDI-TOF: Matrix Assisted Laser Desorption/lonization Time of Flight Mass Spectrometry; MICs: Minimum inhibitory concentrations; SOT: Solid organ transplantation; SPSS: Statistical Package for the Social Sciences; SSTIs: Skin and soft tissue infections; UTIs: Urinary tract infections

\section{Acknowledgements}

Not applicable.

\section{Authors' contributions}

Conceived and designed the experiments: FB, LB, AG. Performed the experiments: LB, FB, GM, CV, MM, SS, SC, AMM. Analyzed the data: LB, FB, RM, MV, GM. Contributed reagents/materials/analysis tools: GM, MM, SS, AMM. Wrote the paper: $L B, F B, G M, M M$. All authors drafted the article, revised it critically for important intellectual content, and approved the final article.

\section{Funding}

Not applicable.

\section{Availability of data and materials}

The data that support the findings of this study are available from Azienda Ospedaliero-Universitaria Ospedali Riuniti Umberto lo-Lancisi-Salesi but restrictions apply to the availability of these data, which were used under license for the current study, and so are not publicly available. Data are however available from the authors upon reasonable request and with permission of the Institutional Review Board of Azienda Ospedaliero-Universitaria Ospedali Riuniti Umberto I-Lancisi-Salesi.

\section{Ethics approval and consent to participate}

The present research has been performed in accordance with the ethical standards of the 1964 Declaration of Helsinki and its later amendements. The Institutional Review Board of the Azienda Ospedaliero-Universitaria Ospedali Riuniti Umberto ${ }^{\circ}$-Lancisi-Salesi granted retrospective access to the data without need for individual informed consent.

\section{Consent for publication}

Not applicable.

\section{Competing interests}

The authors report no competing interests. FB is Associate Editor of BMC Infectious Diseases Journal. The authors alone are responsible for the content and the writing of the paper.

\section{Author details \\ ${ }^{1}$ Clinica Malattie Infettive, Dipartimento di Scienze Biomediche e Sanità Pubblica, Università Politecnica delle Marche, Azienda Ospedaliero-Universitaria Ospedali Riuniti Umberto I-Lancisi-Salesi, Via Conca, 60126 Ancona, Italy. ${ }^{2}$ Unità di Microbiologia, Dipartimento di Scienze Biomediche e Sanità Pubblica, Università Politecnica delle Marche, Via Conca, 60126 Ancona, Italy. ${ }^{3}$ Laboratorio di Microbiologia, Azienda \\ Ospedaliero-Universitaria Ospedali Riuniti Umberto Iº-Lancisi-Salesi, Ancona, Italy. ${ }^{4}$ Chirurgia Epatobiliare e dei Trapianti, Università Politecnica delle Marche, Azienda Ospedaliero-Universitaria, Ospedali Riuniti Umbero ${ }^{\circ}$-Lancisi-Salesi, Ancona, Italy. ${ }^{5}$ Malattie Infettive, Azienda Ospedaliera Ospedali Riuniti Marche Nord, Pesaro, Italy.}

Received: 20 February 2019 Accepted: 8 July 2019 Published online: 12 July 2019

\section{References}

1. Nordmann P, Naas T, Poirel L. Global spread of carbapenemase-producing Enterobacteriaceae. Emerg Infect Dis. 2011;17:1791-8.

2. Tumbarello M, Trecarichi EM, De Rosa FG, Giannella M, Giacobbe DR, Bassetti M, Losito AR, Bartoletti M, Del Bono V, Corcione S, et al. Infections caused by KPC-producing Klebsiella pneumoniae: differences in therapy and mortality in a multicentre study. J Antimicrob Chemother. 2015;70:2133-43.

3. Tumbarello M, Viale $P$, Viscoli $C$, Trecarichi EM, Tumietto F, Marchese A, Spanu T, Ambretti S, Ginocchio F, Cristini F, Losito AR, Tedeschi S, Cauda R, Bassetti M. Predictors of mortality in bloodstream infections caused by Klebsiella pneumoniae carbapenemase-producing $\mathrm{K}$. pneumoniae: importance of combination therapy. Clin Infect Dis. 2012;55:943-50.

4. Daikos GL, Tsaousi S, Tzouvelekis LS, Anyfantis I, Psichogiou M, Argyropoulou A, Stefanou I, Sypsa V, Miriagou V, Nepka M, et al. Carbapenemase-producing Klebsiella pneumoniae bloodstream infections: lowering mortality by antibiotic combination schemes and the role of carbapenems. Antimicrob Agents Chemother. 2014;58: 2322-8.

5. Daikos GL, Markogiannakis A. Carbapenemase-producing Klebsiella pneumoniae: (when) might we still consider treating with carbapenems? Clin Microbiol Infect. 2011;17:1135-41.

6. Qureshi ZA, Paterson DL, Potoski BA, Kilayko MC, Sandovsky G, Sordillo E, Polsky B, Adams-Haduch JM, Doi Y. Treatment outcome of bacteremia due to KPC-producing Klebsiella pneumoniae: superiority of combination antimicrobial regimens. Antimicrob Agents Chemother. 2012;56:2108-13.

7. Sbrana F, Malacarne P, Viaggi B, et al. Carbapenem-sparing antibiotic regimens for infections caused by Klebsiella pneumoniae carbapenemase-producing K. pneumoniae in intensive care unit. Clin Infect Dis. 2013;56:697-700.

8. Hirsch EB, Tam VH. Detection and treatment options for Klebsiella pneumoniae carbapenemases (KPCS): an emerging cause of multidrugresistant infection. J Antimicrob Chemother. 2010;65:1119-25.

9. Falcone M, Russo A, lacovelli A, Restuccia G, Ceccarelli G, Giordano A, Farcomeni A, Morelli A, Venditti M. Predictors of outcome in ICU patients with septic shock caused by Klebsiella pneumoniae carbapenemaseproducing K. pneumoniae. Clin Microbiol Infect. 2016;22:444-50.

10. European Centre for Disease Prevention and Control. Surveillance of antimicrobial resistance in Europe 2016. In: Annual report of the European antimicrobial resistance surveillance network (EARS-Net). Stockholm: ECDC; 2017.

11. Trecarichi EM, Tumbarello M. Therapeutic options for carbapenemresistant Enterobacteriaceae infections. Virulence. 2017;8:470-84.

12. Giacobbe DR, Del Bono V, Trecarichi EM, De Rosa FG, Giannella M, Bassetti M, Bartoloni A, Losito AR, Corcione S, Bartoletti M, Mantengoli E, Saffioti C, Pagani N, Tedeschi S, Spanu T, Rossolini GM, Marchese A, Ambretti S, Cauda R, Viale P, Viscoli C, Tumbarello M, ISGRI-SITA (Italian Study Group on Resistant Infections of the Società Italiana Terapia Antinfettiva). Risk factors for bloodstream infections due to colistin-resistant KPC-producing Klebsiella pneumoniae: results from a multicenter case-control-control study. Clin Microbiol Infect. 2015:21:1106 e1-8.

13. Zarkotou O, Pournaras S, Tselioti P, Dragoumanos V, Pitiriga V, Ranellou K, Prekates A, Themeli-Digalaki K, Tsakris A. Predictors of mortality in patients with bloodstream infections caused by KPC-producing Klebsiella pneumoniae and impact of appropriate antimicrobial treatment. Clin Microbiol Infect. 2011;17:1798-803.

14. Pournaras S, Virioni G, Neou E, Dendrinos J, Dimitroulia E, Poulou A, et al. Predictors of mortality in bloodstream infections caused by Klebsiella pneumoniae carbapenemase (KPC)-producing Enterobacteriacae strains by time-kill assay. Int J Antimicrob Agents. 2011;37:244-7.

15. Rojas LJ. Salim M, Cober E, Richter SS, Perez F, Salata RA, Kalayjian RC, Watkins RR, Marshall S, Rudin SD, Domitrovic TN, Hujer AM, Hujer KM, Doi Y, Kaye KS, Evans S, fowler VG, Bonomo RA, van Duin D, antibacterial resistance leadership group. Colistin resistance in Carbapenem-resistant Klebsiella pneumoniae: laboratory detection and impact on mortality. Clin Infect Dis. 2017:64:711-8.

16. Centers for Disease Control and Prevention (CDC). Bloodstream Infection Event (Central Line-Associated Bloodstream Infection and non-central lineassociated Bloodstream Infection). 2017. (Available from: https://www.cdc. gov/nhsn/pdfs/pscmanual/4psc_clabscurrent.pdf).

17. Yuan M, Aucken H, Hall LM, Pitt TL, Livermore DM. Epidemiological typing of klebsiellae with extended-spectrum beta-lactamases from European intensive care units. J Antimicrob Chemother. 1998;41:527-39.

18. Tenover FC, Arbeit RD, Goering RV, Mickelsen PA, Murray BE, Persing BH, Swaminathan B. Interpreting chromosomal DNA restriction patterns 
produced by pulsed-field gel electrophoresis: criteria for bacterial strain typing. J Clin Microbiol. 1995;33:2233-9.

19. Diancourt L, Passet V, Verhoef J, Grimont PA, Brisse S. Multilocus sequence typing of Klebsiella pneumoniae nosocomial isolates. J Clin Microbiol. 2005; 43:4178-82.

20. The European Committee on Antimicrobial Susceptibility Testing. Breakpoint tables for interpretation of MICs and zone diameters. Version 8.0, 2018. http://www.eucast.org/fileadmin/src/media/PDFs/EUCAST_files/ Breakpoint_tables/v_8.0_Breakpoint_Tables.pdf.

21. Del Bono V, Giacobbe DR, Marchese A, Parisini A, Fucile C, Coppo E, Marini V, Arena A, Molin A, Martelli A, Gratarola A, Viscoli C, Pelosi P, Mattioli F. Meropenem for treating KPC-producing Klebsiella pneumoniae bloodstream infections: should we get to the PK/PD root of the paradox? Virulence. 2017:8:66-73.

22. Pea F, Della Siega P, Cojutti P, Sartor A, Crapis M, Scarparo C, Bassetti M. Might real-time pharmacokinetic/pharmacodynamic optimisationof highdose continuous-infusion meropenem improve clinical cure ininfections caused by KPC-producing Klebsiella pneumoniae? Int J Antimicrob Agents. 2017;49:255-8

23. Gomez-Simmonds A, Nelson B, Eiras DP, Loo A, Jenkins SG, Whittier S, Calfee DP, Satlin MJ, Kubin CJ, Furuya EY. Combination regimens for treatment of Carbapenem-resistant Klebsiella pneumoniae bloodstream infections. Antimicrob Agents Chemother. 2016;60:3601-7.

24. Conte V, Monaco M, Giani T, D'Ancona F, Moro ML, Arena F, D'Andrea MM, Rossolini GM. Pantosti a; AR-ISS study group on Carbapenemase-Producing K. pneumoniae. Molecular epidemiology of KPC-producing Klebsiella pneumoniae from invasive infections in Italy: increasing diversity with predominance of the ST512 clade II sublineage. J Antimicrob Chemother. 2016;71:3386-91.

25. Villa L, Feudi C, Fortini D, Brisse S, Passet V, Bonura C, Endimiani A Mammina C, Ocampo AM, Jimenez JN, Doumith M, Woodford N, Hopkins K, Carattoli A. Diversity, virulence, and antimicrobial resistance of the KPCproducing Klebsiella pneumoniae ST307 clone. Microb Genom. 2017;3: e000110.

\section{Publisher's Note}

Springer Nature remains neutral with regard to jurisdictional claims in published maps and institutional affiliations.

Ready to submit your research? Choose BMC and benefit from:

- fast, convenient online submission

- thorough peer review by experienced researchers in your field

- rapid publication on acceptance

- support for research data, including large and complex data types

- gold Open Access which fosters wider collaboration and increased citations

- maximum visibility for your research: over $100 \mathrm{M}$ website views per year

At $\mathrm{BMC}$, research is always in progress.

Learn more biomedcentral.com/submissions 